\title{
In vitro effects of erosive challenge on the surface properties of sealants
}

\author{
Isabella Cavalcante Medeiros ${ }^{1}$, Bruna Palmeira Costa ${ }^{1}$, Brenna Louise Cavalcanti Gondim ${ }^{1}$, \\ Hugo Lemes Carlo ${ }^{1}$, Rogério Lacerda dos Santos ${ }^{1}$, Fabíola Galbiatti de Carvalho ${ }^{1}$
}

${ }^{1}$ Universidade Federal da Paraíba - UFPB, Health Science Center, Department of Clinical and Social Dentistry, João Pessoa, PB, Brazil

Received for publication: November 18, 2015 Accepted: December 12, 2015

Correspondence to: Fabíola Galbiatti de Carvalho Universidade Federal da Paraíba - Centro de Ciências da Saúde - Programa de Pós-Graduação em Odontologia - Campus I CEP: 58051-900 - João Pessoa, Paraíba, Brasil Phone: +55 8332167251 Fax:+55 8332167797

E-mail: fabigalbi@yahoo.com.br

\section{Abstract}

Aim: To assess in vitro the surface roughness (Ra), Vickers hardness (VHN) and surface morphology of resin and glass ionomer materials used for sealants after dynamic erosive challenge. Methods: Twenty specimens of each material were prepared and divided into experimental (erosive challenge) and control groups ( $n=10)$ : Protect Riva (SDI), Opallis Flow (3M ESPE), Fluroshield (Dentsply), Filtek Z350 XT Flow (3M ESPE). The erosive challenge was performed 4 times per day (90 s) in cola drink and for $2 \mathrm{~h}$ in artificial saliva for 7 days. The control specimens were maintained in artificial saliva. Ra and VHN readings were performed before and after erosion. The percentage of hardness loss (\%VHN) was obtained after erosion. The surface morphology was evaluated by scanning electron microscopy (SEM). The data were analyzed by ANOVA, Tukey and paired t tests $(\alpha=0.05)$. Results: After erosion and saliva immersion, there was an increase in Ra values for all groups and Riva group showed the highest Ra values. After erosive challenge, Riva and Filtek groups showed significant decrease in VHN values, but Filtek group showed the greatest \%VHN. For all groups there was inorganic particle protrusion and matrix degradation after erosion visualized by SEM images. Conclusions: Erosive challenge affected the surface properties of all materials used as sealants, particularly in the Riva and Filtek groups.

Keywords: tooth erosion; composite resins; glass ionomer cements; hardness tests.

\section{Introduction}

Dental erosion is defined as the loss of tooth substance by the chemical process of acid exposure and dissolution, involving no bacterial plaque acid ${ }^{1}$. The acids in food and drink are considered the major etiological factors responsible for erosive lesions in enamel ${ }^{1-2}$. There is evidence that the prevalence of erosion is increasing because of the high consumption of soft drinks ${ }^{3}$.

The most important aspects in the treatment of patients with dental erosion are diagnosis and prevention of lesion progression to limit additional destruction of tooth tissue. Elimination of the acid source and preventive approaches using fluoride compounds are indicated to control the tooth erosion or the dental tissue softening caused by acidic solutions ${ }^{4-7}$.

The effects of dentin-bonding agents and restorative materials used to seal dental structures have also been advocated to control or prevent the development of erosion lesions ${ }^{8-10}$. It was shown that resin-based bonding agents could protect against erosion and abrasion in situ ${ }^{8,10}$. Wegehaupt et al. ${ }^{11}(2012)$ demonstrated that resin-based materials were also able to reduce the erosive demineralization of bovine enamel after immersion in hydrochloric and citric acids over consecutive days. However, to simulate what occurs in the oral cavity, in vitro studies used 
dynamic erosive $\mathrm{pH}$-cycling challenge, with daily cycles of immersion in acid solution and artificial saliva, to mimic the daily ingestion of acidic beverages ${ }^{6,12}$.

It would be interesting to investigate the surface properties of materials used as sealants after erosive challenge because the surface properties of sealant materials can be used to evaluate the surface degradation of the material and to predict its resistance in the oral environment. However, few studies have evaluated the surface properties of materials used as sealants after dynamic erosive challenge ${ }^{12}$.

Thus, the aim of this study was to evaluate the surface microhardness and roughness of ionomeric and resin-based materials used as sealants and their morphological surface characteristics using scanning electron microscopy (SEM), after erosive challenge with a cola beverage. The tested hypothesis was that there would be differences in microhardness, roughness and morphological surface characteristics among the sealants after erosive challenge.

\section{Material and methods}

\section{Specimen preparation}

Four materials used as sealants were investigated in this study: three resin-based sealants - Filtek Z350 XT Flow (3M/ ESPE, St. Paul, MN, USA), Fluroshield (Dentsply, Rio de Janeiro, RJ, Brazil) and Opallis Flow (FGM, Joinville, SC, Brazil); and one glass ionomer sealant - Riva Protect (SDI, Bayswater, Victoria, Australia). The compositions of the evaluated materials are in Table 1.

Twenty specimens of each material were fabricated using silicone molds ( $4 \mathrm{~mm}$ diameter $\times 2 \mathrm{~mm}$ high), according to the manufacturers' instructions. The capsules of Riva Protect were triturated for $10 \mathrm{~s}$ in an amalgamator (Ultramat 2, SDI, Bayswater, Victoria, Australia). Then, the material was inserted into the matrix with the Riva applicator. The specimens were covered with acetate strips (Probem Ltda, Catanduva, São Paulo, Brazil) and were pressed flat with a glass slide for 5 min to obtain a smooth surface. The resin-based materials were inserted into the matrix and were covered with acetate strips and polymerized for $20 \mathrm{~s}$ with a LED curing light (1200 $\mathrm{mW} / \mathrm{cm}^{2}$ - Radii Cal; SDI). The specimens were maintained in relative humidity for $24 \mathrm{~h}$, before the baseline roughness and microhardness measurements were obtained as described above. Specimens of each material were divided in two groups $(\mathrm{n}=10)$ : erosion (erosive challenge) and control (artificial saliva immersion) groups.

\section{Dynamic erosive $\mathrm{pH}$-cycling challenge}

The specimens were immersed in cola drink (Coca-Cola ${ }^{\circledR}$, $\mathrm{SP}$, Brazil - $\mathrm{pH} 2.3$ ) at room temperature in individual containers $(10 \mathrm{~mL} / \mathrm{specimen})$ for $90 \mathrm{~s}$ four times/day ${ }^{4-5}$. Subsequently, the specimens were rinsed thoroughly with deionized water and were immersed in artificial saliva with a $7.0 \mathrm{pH}(10 \mathrm{~mL} / \mathrm{block})$ at room temperature for $2 \mathrm{~h}$, both between erosive challenges and overnight ${ }^{4-5}$. The artificial saliva was fabricated according to Carvalho et al. ${ }^{13}$ (2013). This erosive challenge was repeated for 7 days. The cola drink and artificial saliva were changed after every cycle. During the acidic cycles, the samples were kept in hermetically sealed containers to prevent the loss of carbonation from the cola drink. The specimens in the control group were immersed in artificial saliva for 7 days. The artificial saliva was changed every day.

\section{Surface roughness measurements}

At the end of the erosive challenge, the specimens were ultrasonically washed for $10 \mathrm{~min}$ and dried with absorbent paper. They were then fitted to a surface roughness-measuring instrument (TR200, Digimess, São Paulo, SP, Brazil). In each specimen, three successive measurements in the central area

Table 1 - Compositions of materials used as sealants in the study.

\begin{tabular}{|c|c|c|}
\hline Sealant/Batch number & Type & Composition* \\
\hline $\begin{array}{l}\text { Riva Protect } \\
\text { (SDI, Bayswater, Victoria, } \\
\text { Australia, F1111031) }\end{array}$ & Glass ionomer & $\begin{array}{l}\text { Capsule: Compartment } 1 \text { (powder): Fluoro aluminosilicate glass (90\% load size } \\
\text { not specified) and polyacrylic acid (10\%); Compartment } 2 \text { (liquid): polyacrylic } \\
\text { acid (25\%), tartaric acid (10\%) and balanced ingredient (non-hazardous) }(65 \%)\end{array}$ \\
\hline $\begin{array}{l}\text { Fluroshield } \\
\text { (Dentsply, Rio de Janeiro, } \\
\text { RJ, Brazil, 946229G) }\end{array}$ & Resin & $\begin{array}{c}\text { UED-Bis-GMA }(<40 \%) \text {, barium aluminoborosilicate glass }(30 \%) \text {, polymerizable } \\
\text { dimethacrylate resin }(<10 \%) \text {, Bis-GMA }(<5 \%) \text {, sodium fluoride }(<5 \%) \text {, } \\
\text { dipentaerythritol pentaacrylate phosphate }(<5 \%) \text {, titanium dioxide }(<3 \%), \\
\text { amorphous silica }(<2 \% \text { load size not specified) }\end{array}$ \\
\hline $\begin{array}{l}\text { Filtek Z350 XT } \\
\text { (3MESPE, St. Paul, MN, } \\
\text { USA, N385003) }\end{array}$ & Resin & $\begin{array}{l}\text { Silane-treated ceramic (52-60\%), Bis-GMA (10-15\%), TEGDMA (10-15\%), Bis- } \\
\text { EMA (1-5\%), silane-treated silica (3-11\%), silane-treated zirconium oxide (3-11\% } \\
\text { mean load size 0.01-6 } \mu \mathrm{m}) \text {, functionalized dimethacrylate polymer (1-5\%) }\end{array}$ \\
\hline $\begin{array}{l}\text { Opallis Flow } \\
\text { (FGM, Joinville, SC, Brazil, } \\
\text { 040612) }\end{array}$ & Resin & $\begin{array}{l}\text { UDMA (5-10\%), TEGDMA (5-10\%), Bis-EMA (5-10\%), silanized inorganic filler, } \\
\text { Ba-Al-Si micro-particles and } \mathrm{SiO}_{2} \text { in nanoparticles }(0.05 \text { and } 5.0 \mu \mathrm{m})(\sim 72 \%)\end{array}$ \\
\hline
\end{tabular}


in different directions were performed by the same examiner, and the mean surface roughness values (Ra) were obtained and expressed in micrometers. The roughness test was performed at baseline and $24 \mathrm{~h}$ after erosive challenge.

\section{Surface Vickers microhardness}

The microhardness measurements were obtained with a hardness tester (HMV II; Shimadzu Corporation, Kyoto, Japan), using a Vickers indenter (VHN) and a $200 \mathrm{~g}$ load, with a $15 \mathrm{~s}$ dwell time ${ }^{14}$. Five indentations were made in each specimen, at least $50 \mu \mathrm{m}$ apart, and the mean VHN value was obtained. In addition, the percentage of microhardness loss $(\% \mathrm{VHN})$ was calculated using the following formula ${ }^{12}$ :

$\% \mathrm{VHN}=100\left(\mathrm{VHN}_{(\mathrm{F})}-\mathrm{VHN}_{(\mathrm{I})}\right) / \mathrm{VHN}_{(\mathrm{I})}$,

where $\mathrm{VHN}_{(\mathrm{I})}$ is the average of the initial (baseline) microhardness measurements, and $\mathrm{VHN}_{(\mathrm{F})}$ is the average of the ûnal (after erosive challenge) microhardness values.

\section{Scanning electron microscopy (SEM)}

Three representative specimens from each group were mounted on aluminum stubs and sputter-coated with gold in vacuum (Balzers-SCD 050 Sputter Coater, Balzers, Liechtenstein). Three extra specimens were prepared for baseline (without saliva or coca immersion) evaluation. A LEO 1430 scanning electron microscope (Zeiss Inc., Thornwood, NY, USA) was used for SEM analyses. Analyses were performed at $1500 \mathrm{X}$ and $2000 \mathrm{X}$ magnification before and after the erosive challenge and immersion in artificial saliva.

\section{Statistical analysis}

The data were analyzed using GraphPad Instat software, version 2.0 (GraphPad Software, La Jolla, CA, USA) at a significance level $\alpha=0.05$. The sample size was calculated considering the minimum difference between the average of treatments (mean \pm standard deviation) of $0.5 \pm 0.05 \mu \mathrm{m}$ for roughness testing and $13.0 \pm 3.0 \mathrm{VHN}$ for microhardness testing. With a significance level of 0.05 and a power of $95 \%$, a minimum of four specimens per group was required. All tested variables satisfied the assumptions of normal distribution, therefore two-way ANOVA and Tukey's test were performed for statistical comparisons of Ra and VHN measurements among the sealants. Student's paired t-test was used compare Ra and VHN measurements before and after erosive challenge for the same sealant. Unpaired t-test was used to compare Ra and VHN values after erosive challenge and artificial saliva immersion for each sealant.

\section{Results}

The results of the roughness and microhardness tests are in Tables 2 and 3, respectively. When the Ra values between baseline and post-treatment (after erosion or after saliva) were compared, there was a statistically significant increase in $\mathrm{Ra}$ values in all the groups $(\mathrm{p}=0.001)$, except for the Fluroshield and Filtek groups, which did not show significant differences in Ra values after artificial saliva immersion ( $p=0.06$ and $p=0.08$, respectively) (Table 2 ). When the comparison was made among sealants after erosion

Table 2 - Surface roughness (Ra) of the sealant materials after erosive challenge and immersion in artificial saliva (control). Values are expressed as means \pm standard deviations $(\mu \mathrm{m})$.

\begin{tabular}{lllll}
\hline \multirow{2}{*}{ Sealants } & \multicolumn{4}{c}{ Roughness values (Ra) } \\
\cline { 2 - 5 } & \multicolumn{2}{c}{ Erosion group } & \multicolumn{2}{c}{ Artificial saliva group } \\
\cline { 2 - 5 } Riva Protect & Baseline & After erosion & Baseline & After saliva \\
Opallis Flow & $0.29 \pm 0.07^{\mathrm{A}, \mathrm{a}^{\mathrm{a}}}$ & $0.45 \pm 0.06^{\mathrm{B}, \mathrm{a}^{\mathrm{a}}}$ & $0.29 \pm 0.03^{\mathrm{A}, \mathrm{a}}$ & $0.35 \pm 0.02^{\mathrm{B}, \mathrm{a}}$ \\
Filtek Z350 XT & $0.04 \pm 0.01^{\mathrm{A}, \mathrm{b}}$ & $0.07 \pm 0.01^{\mathrm{B}, \mathrm{b}}$ & $0.04 \pm 0.01^{\mathrm{A}, \mathrm{b}}$ & $0.06 \pm 0.01^{\mathrm{B}, \mathrm{b}}$ \\
Fluroshield & $0.06 \pm 0.02^{\mathrm{A}, \mathrm{b}}$ & $0.10 \pm 0.02^{\mathrm{B}, \mathrm{b}}$ & $0.07 \pm 0.03^{\mathrm{A}, \mathrm{b}}$ & $0.06 \pm 0.01^{\mathrm{A}, \mathrm{b}}$ \\
\hline
\end{tabular}

* The same uppercase letters indicate that there was no significant difference between the initial and post-treatment values of each sealant material (paired t test, $p>0.05$ ).

** The same lowercase letters indicate that there were no significant differences among the sealants materials at baseline or after treatment (two-way ANOVA and Tukey's test, $p>0.05$ ).

Table 3 - Surface microhardness (VHN) and percentage of VHN loss of sealant materials after erosive challenge and immersion in artificial saliva (control). Values expressed as means \pm standard deviations

\begin{tabular}{|c|c|c|c|c|c|c|}
\hline \multirow{3}{*}{ Sealants } & \multicolumn{4}{|c|}{ Microhardness values (VHN) } & & \\
\hline & & Erosion grou & & \multicolumn{3}{|c|}{ Artificial saliva group } \\
\hline & Baseline & After erosion & $\%$ VHN loss & Baseline & After saliva & \%VHN loss \\
\hline Opallis Flow & $43.5 \pm 2.6^{A}$ & $40.2 \pm 1.9^{A}$ & $-7.6^{b}$ & $41.5 \pm 3.1^{A}$ & $39.6 \pm 2.1^{A}$ & $-7.9^{a}$ \\
\hline Filtek Z350 XT & $44.1 \pm 0.7^{A}$ & $24.1 \pm 0.9^{B}$ & $-44.6^{a}$ & $43.3 \pm 0.9^{A}$ & $43.1 \pm 1.2^{\mathrm{A}}$ & $-2.7^{\mathrm{a}}$ \\
\hline Fluroshield & $21.4 \pm 2.6^{A}$ & $20.4 \pm 2.4^{\mathrm{A}}$ & $-5.6^{b}$ & $20.3 \pm 1.6^{\mathrm{A}}$ & $19.9 \pm 1.9^{A}$ & $-6.0^{\mathrm{a}}$ \\
\hline
\end{tabular}

*The same uppercase letters indicate that there was no significant difference between the initial and post-treatment values of each sealant material (paired t test, $\mathrm{p}>0.05)$.

**The same lowercase letters indicate that there were no significant differences among sealant materials at baseline or after treatment (two-way ANOVA and Tukey's test, $p>0.05)$. 
and after saliva immersion, the Riva group showed the highest Ra values $(p=0.001)$ and there were no significant differences among other groups after both treatments $(p=0.07)$ (Table 2).

After erosive challenge, the Riva and Filtek groups showed significant decrease in VHN values $(p=0.03$ and $p=0.001$, respectively), but after saliva immersion, there were no significant VHN alterations in any group (Table 3). After erosion, when the \%VHN values were compared, the Filtek group had the greatest value $(p=0.001)$ and Riva, Fluroshield and Opallis groups did not show significant differences among them $(p=0.08)$. However, after artificial saliva immersion, there were no significant differences in \% VHN among the groups $(\mathrm{p}=0.10)($ Table 3$)$.

Tables 4 and 5 show the comparison of Ra and VHN values of each material between "after erosion" and "after saliva immersion" treatments, respectively. For all the materials, the erosion treatment showed significantly higher Ra values when compared to saliva immersion treatment, except for the Opallis group $(p=0.08)$ (Table 4). For VHN measurements, Riva and Filtek groups showed higher VHN values after saliva immersion compared to after erosion treatment $(p=0.03$ and $p=0.001$, respectively) (Table 5).

Figures 1, 2, 3 and 4 show the SEM images at baseline, after erosive challenge and after saliva immersion. For all the groups, after the erosive challenge, a protrusion of the inorganic particles and degradation of the ionomeric or resin matrix (Figures 1B, 2B, 3B and 4B) was observed. The Riva group had greater surface degradation than the other groups, showing a porous surface and matrix deterioration (Figure 1B). The cracks visualized on ionomeric sealant images

Table 4 - Surface roughness (Ra) measurements of sealant materials after erosive challenge and artificial saliva immersion. Values are expressed as mean \pm standard deviation.

\begin{tabular}{lll}
\hline Sealants & \multicolumn{2}{c}{ Roughness values $(\mathrm{Ra})(\mu \mathrm{m})$} \\
\cline { 2 - 3 } & After erosion & After saliva \\
Riva Protect & $0.45 \pm 0.06^{\mathrm{A}^{*}}$ & $0.35 \pm 0.02^{\mathrm{B}}$ \\
Opallis Flow & $0.07 \pm 0.01^{\mathrm{A}}$ & $0.06 \pm 0.01^{\mathrm{A}}$ \\
Filtek Z350 XT & $0.10 \pm 0.02^{\mathrm{A}}$ & $0.06 \pm 0.01^{\mathrm{B}}$ \\
Fluroshield & $0.13 \pm 0.02^{\mathrm{A}}$ & $0.08 \pm 0.01^{\mathrm{B}}$ \\
\hline
\end{tabular}

* The same uppercase letters indicate that there was no significant difference in Ra values between the erosion and saliva treatments of each sealant material (unpaired t test, $p>0.05)$.

Table 5 - Microhardness (VHN) measurements of sealant materials after erosive challenge and artificial saliva immersion. Values are expressed as mean \pm standard deviation.

\begin{tabular}{lll}
\hline Sealants & \multicolumn{2}{c}{ Microhardness values (VHN) } \\
\cline { 2 - 3 } & After erosion & After saliva \\
Riva Protect & $59.4 \pm 3.3^{\mathrm{B}^{\mathrm{A}}}$ & $64.0 \pm 2.4^{\mathrm{A}}$ \\
Opallis Flow & $40.2 \pm 1.9^{\mathrm{A}}$ & $39.6 \pm 2.1^{\mathrm{A}}$ \\
Filtek Z350 XT & $24.1 \pm 0.9^{\mathrm{B}}$ & $43.1 \pm 1.2^{\mathrm{A}}$ \\
Fluroshield & $20.4 \pm 2.4^{\mathrm{A}}$ & $19.9 \pm 1.9^{\mathrm{A}}$ \\
\hline
\end{tabular}

* The same uppercase letters indicate that there was no significant difference in $\mathrm{VHN}$ values between the erosion and saliva treatments of each sealant material (unpaired t test, $p>0.05$ ).
(Figure 1) were artifacts caused by vacuum during sample preparation. After artificial saliva immersion, slight surface degradation was observed in the Riva and Fluroshield groups (Figures 1C and 2C), but the other groups did not show morphological differences between baseline and the treatments. Furthermore, the Opallis group showed the smallest surface morphological changes among baseline, erosive challenge and artificial saliva immersion (Figure 4).

\section{Discussion}

Resin and ionomeric materials can be used in clinical practice to seal tooth structures and to prevent dental erosion $^{8,11}$. It is known that, during consumption, beverages contact only with the tooth surface and restorative materials for a short time before they are washed away by saliva ${ }^{15}$. In previous studies, the sealant surfaces have usually contacted acidic beverages for prolonged periods of time, or the studies did not include the saliva in their methodologies ${ }^{11,16}$. The

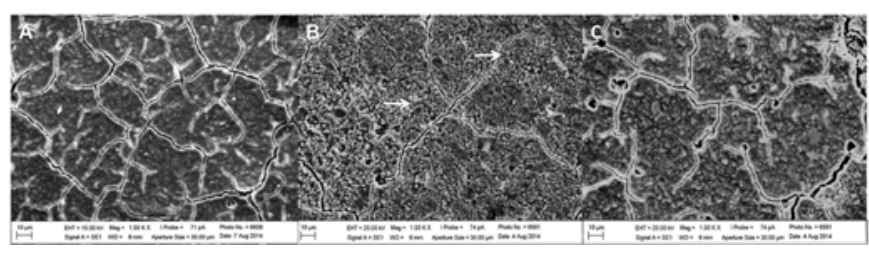

Fig.1. SEM images of the surface morphology for the Riva Protect group (1500 X): (A) baseline; (B) after erosive challenge and (C) after saliva immersion; $(\rightarrow)$ : filler particles.

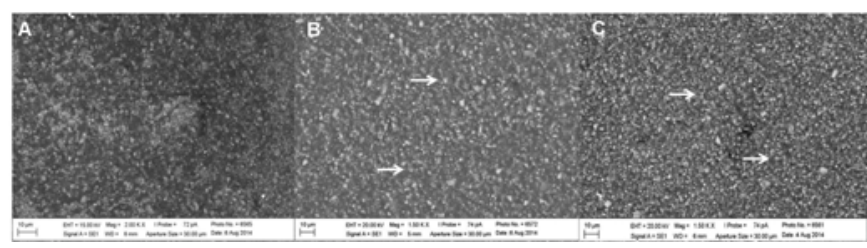

Fig. 2. SEM images of the surface morphology for the Fluroshield group $(1500 \mathrm{X})$ : (A) baseline; (B) after erosive challenge and (C) after saliva immersion; $(\rightarrow)$ : filler particles.

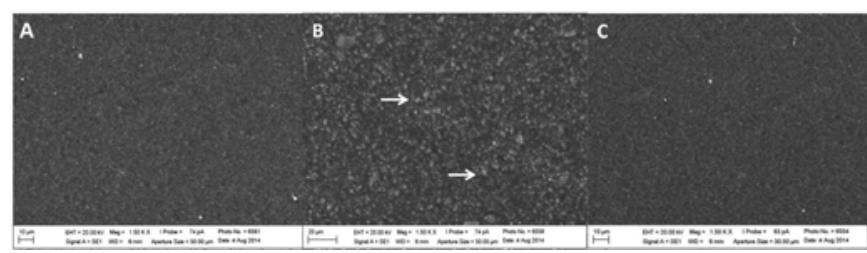

Fig. 3. SEM images of the surface morphology for the Filtek Z350XT Flow group $(1500 \mathrm{X})$ : (A) baseline; (B) after erosive challenge and (C) after saliva immersion; $(\rightarrow)$ : filler particles.

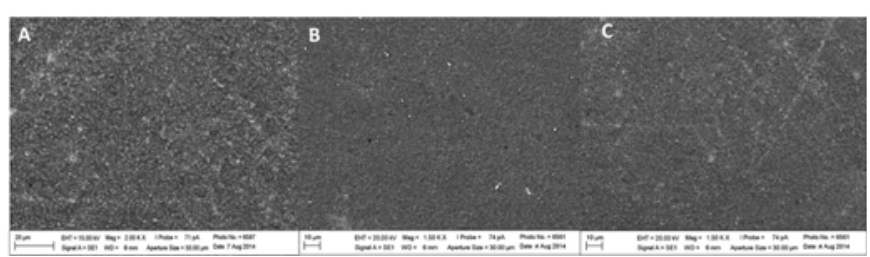

Fig. 4. SEM images of the surface morphology for the Opallis flow group: (A) baseline (2000X); (B) after erosive challenge (1500 X) and (C) after saliva immersion $(1500 \mathrm{X})$. 
present study was designed to overcome these limitations of in vitro erosion studies, using a dynamic erosive $\mathrm{pH}$-cycling model. This dynamic erosive model simulated the typical consumption of individuals considered to be at risk for dental erosion $^{4-6}$, using a beverage (Coca-Cola, $\mathrm{pH} 2.3$ ) that is widely consumed by the population and that has high erosive potential due to its low $\mathrm{pH}$ and fluoride/calcium concentrations ${ }^{2}$.

The results showed an increase in $\mathrm{Ra}$ values for all of the sealants after the erosive challenge (Table 2). Francisconi et al. ${ }^{12}$ (2008) found wear of approximately $0.3 \mu \mathrm{m}$ for resin and glass ionomer restorative materials after an erosive challenge similar to that used in the present study. According to these authors, roughness values of materials after erosive challenge of approximately 0.1-0.4 $\mu \mathrm{m}$ could be considered low, like the Ra values in the present study. For resin-based sealant materials, the phosphoric acid found in cola beverage could induce softening of the bisphenol-A-glycidyl methacrylate (Bis-GMA) polymers in resin sealants, which could result from the leaching of diluent agents, such as triethylene glycol dimethacrylate (TEGDMA) ${ }^{12}$. Additionally, softening of the resin matrix could favor the displacement of inorganic fillers, contributing to the formation of a rough surface.

The ionomeric sealant (Riva group) showed the highest $\mathrm{Ra}$ values before and after erosion and artificial saliva immersion (Table 2). SEM images also showed greater surface degradation of in the Riva group than in other groups, with a porous surface and higher matrix deterioration (Figure 1B). Because the size of the glass filler particles in ionomeric cements is larger than in resin materials ${ }^{17}$ (Figure 1), there is likely less homogeneity between the filler and matrix, thus increasing its surface roughness in both immersion media. Furthermore, in glass ionomer cements, there is microcrack formation in a ionic-crosslinked polyalkenoate matrix, leading to subsequent loss of particle adherence, which could also cause increases in roughness and wear ${ }^{17}$. Similar to resinbase sealants, acidic beverages could dissolve the siliceous hydrogel layer and the matrix peripheral to the glass particles in ionomeric sealant, causing a rougher surface.

The dissolution of siliceous hydrogel matrix could also explain the significant decrease in VHN values in the Riva group compared to other groups after the erosive challenge (Table 3). The study by Francisconi et al. ${ }^{12}$ (2008) also showed that glass ionomer cements had greater microhardness losses compared to resin composites, because the acid attack on the resin matrix occurred in a lesser extent than in the siliceous hydrogel matrix.

After artificial saliva immersion, there were no significant differences among the sealants in \%VHN loss; it seems that the acidic $\mathrm{pH}$ of beverages could interfere to a greater extent in the organic matrix degradation of the tested materials. In the present study, the Filtek group showed the greatest \%VHN loss, compared to other resin-based sealants after erosive challenge (Table 3).

It is known that inorganic filler particles reduce polymerization shrinkage at the same time as they enhance the mechanical properties of the resin material ${ }^{18}$. The higher percentage of inorganic filler $(72 \%)$ and the lower organic content $(30 \%)$ of Opallis sealant, compared with Filtek Z350 XT $(52-60 \%$ and $3-11 \%$, respectively) (Table 1) probably resulted in a smaller $\%$ VHN loss in the Opallis group ${ }^{19}$. The higher percentage of inorganic filler and its possible displacement due to matrix degradation could also explain the higher Ra values after saliva immersion for Opallis group. Furthermore, the percentage of ethoxylated bisphenol-A dimethacrylate (Bis-EMA) in the organic matrix of Opallis was higher than in the Filtek group. The decreased flexibility and elimination of the hydroxyl groups from the Bis-GMA monomer to Bis-EMA increased the hydrophobicity of BisEMA monomer ${ }^{20}$. This characteristic reduced the water uptake by the matrix and its plasticization after contact with oral liquids. Thus, the percentage of Bis-EMA monomer may be partially responsible for the biochemical stability of Opallis sealant in aqueous environments, regardless the $\mathrm{pH}$ of the solution., The Opallis group was the only one that showed no significant differences in $\mathrm{Ra}$ or VHN values after erosive challenge and immersion in artificial saliva (Tables 4 and 5). After erosion, SEM images also showed a surface with matrix degradation and the protrusion of inorganic filler in the Fluroshield and Filtek groups (Figures 2B and 3B, respectively), whereas the Opallis group showed a homogeneous surface similar to that at baseline (Figures $4 \mathrm{~A}$ and $\mathrm{B}$ ). The concentration and particle size of the glass ionomer cement is greater than that of the resin sealants. Thus, it was possible to visualize more easily the matrix degradation and the protrusion of the particles after erosive challenge. A limitation of this study was not showing a higher magnification of SEM images of resin sealants, but even in $1500 \mathrm{x}$ magnification it was still possible to verify the same degradation characteristics (protrusion of the particles) after erosion in Figures 2B and 3B related to Fluroshield and Filtek Z350 materials. For Opallis group (Figure 4) it was not possible to verify the degradation on the surface because this material suffered less degradation, as explained before.

Hardness is a physical property possibly related to the degree of conversion and to the amount of filler particles in resin-based materials ${ }^{21}$. Thus, it is likely that the relatively high degree of conversion of the Bis-GMA/UED-Bis-GMA resin matrix for Fluroshield might have compensated for the small percentage of filler particles, resulting in a smaller $\%$ VHN loss than in the Filtek group (Table 3). It is generally accepted that crosslinked polymers are more resistant to degradation and solvent uptake in aqueous environments, whereas linear polymers present more spaces and pathways for molecules to diffuse within their structures and to degrade the material ${ }^{21}$. These facts may also be the reasons why the Fluroshield group did not show significant differences between VHN values before and after erosive challenge and saliva immersion (Tables 3 and 5). For the other groups, except for Opallis, the erosive challenge caused greater degradation of the surface properties than artificial saliva immersion (Tables 4 and 5). SEM images also showed similarities between the surface morphology after erosion and after saliva immersion for the Fluroshield and Opallis 
groups (Figures 2B, 2C and 4B, 4C).

The oral cavity is the adequate environment for predicting the behavior of dental materials, but in vitro models are very important for providing insight into the fundamental mechanisms of biodegradation ${ }^{12}$. Clinically, a thin layer of sealant is applied on tooth structure to prevent against erosion. This study used specimens $2 \mathrm{~mm}$ thick, but the analysis was restricted to surface properties and the thickness of specimens did not interfere in the results.

Although sealants materials may degrade with time ${ }^{9}$, it may have a role in prevention of tooth erosion and may be a less patient-dependent approach compared to fluoride application, because it does not depend on the patient compliance. In general, the present study showed that that erosive challenge with cola beverage caused changes in the surface properties of sealant materials, and the Opallis group had a better in vitro performance related to surface properties. The hypothesis tested in the present study was accepted because there were differences among the sealants in microhardness, roughness or morphological surface characteristics after erosive challenge. Longer periods of erosive challenge should be used, and other important properties of the sealants, such as adhesion and microleakage, should be studied under erosive challenge.

\section{Acknowledgements}

The authors are grateful to Laisa Daniel Gondim for contributions to the roughness surface analysis and to Professor Severino Jackson Guedes de Lima and Isaque Jerônimo Porto of Rapid Solidification Laboratory/Federal University of Paraíba (LSR/UFPB) for use of SEM.

\section{References}

1. LussiA, Carvalho TS. Erosive tooth wear: a multifactorial condition of growing concern and increasing knowledge. Monogr Oral Sci. 2014; 25: 1-15.

2. Salas MMS, Dantas RVF, Sarmento HR, Vargas-Ferreira F, Torriani D, Demarco FF. Tooth erosion and dental caries in schoolchildren: is there a relationship between them? Braz J Oral Sci. 2014; 13: 12-6.

3. Gurgel CV, Rios D, Buzalaf MA, da Silva SM, Araújo JJ, Pauletto AR, et al. Dental erosion in a group of 12- and 16-year-old Brazilian schoolchildren. Pediatr Dent. 2011; 33: 23-8.

4. Levy FM, Magalhães AC, Gomes MF, Comar LP, Rios D, Buzalaf MA. The erosion and abrasion-inhibiting effect of TiF4 and NaF varnishes and solutions on enamel in vitro. Int J Paediatr Dent. 2012; 22: 11-6.

5. Magalhães AC, Levy FM, Rios D, Buzalaf MA. Effect of a single application of TiF4 and NaF varnishes and solutions on dentin erosion in vitro. J Dent. 2010; 38: 153-7.

6. Medeiros IC, Brasil VL, Carlo HL, Santos RL, De Lima BA, Carvalho FG. In vitro effect of calcium nanophosphate and high-concentrated fluoride agents on enamel erosion: an AFM study. Int J Paediatr Dent. 2014; 24: 168-74.

7. Huysmans MC, Young A, Ganss C. The role of fluoride in erosion therapy. Monogr Oral Sci. 2014; 25: 230-43.

8. Azzopardi A, Bartlett DW, Watson TF, Sherriff M. The surface effects of erosion and abrasion on dentine with and without a protective layer. Br Dent J. 2004; 196: 351-4.

9. Bartlett D, Sundaram G, Moazzez R. Trial of protective effect of fissure sealants, in vivo, on the palatal surfaces of anterior teeth, in patients suffering from erosion. J Dent. 2011; 39: 26-9.
10. Sundaram $G$, Watson T, Bartlett D. Clinical measurement of palatal tooth wear following coating by a resin sealing system. Oper Dent. 2007; 32 539-43.

11. Wegehaupt FJ, Tauböck TT, Sener B, Attin T. Long-term protective effect of surface sealants against erosive wear by intrinsic and extrinsic acids. $J$ Dent. 2012; 40: 416-22.

12. Francisconi LF, Honório HM, Rios D, Magalhães AC, Machado MA, Buzalaf MA. Effect of erosive $\mathrm{pH}$ cycling on different restorative materials and on enamel restored with these materials. Oper Dent. 2008; 33: 203-8.

13. Carvalho FG, Brasil VL, Silva Filho TJ, Carlo HL, Santos RL, Lima BA Protective effect of calcium nanophosphate and CPP-ACP agents on ename erosion. Braz Oral Res. 2013; 27:463-70.

14. Fúcio SB, Carvalho FG, Sobrinho LC, Sinhoreti MA, Puppin-Rontani RM The influence of 30-day-old Streptococcus mutans biofilm on the surface of esthetic restorative materials: an in vitro study. J Dent. 2008; 36:833-9.

15. Honório HM, Rios D, Francisconi LF, Magalhães AC, Machado MA Buzalaf MA. Effect of prolonged erosive $\mathrm{pH}$ cycling on different restorative materials. J Oral Rehabil. 2008; 35: 947-53.

16. Fatima N, Abidi SYA, Qazi F, JatAS. Effect of different Tetra Pack juices on microhardness of direct tooth colored-restorative materials. Saudi Dent J. 2013; 25: 29-32.

17. de Fucio SB, de Paula AB, de Carvalho FG, Feitosa VP, Ambrosano GM, Puppin-Rontani RM. Biomechanical degradation of the nano-filled resinmodified glass-ionomer surface. Am J Dent. 2012; 25: 315-20.

18. Furuse AY, Mondelli J, Watts DC. Network structures of Bis-GMA/TEGDMA resins differ in DC, shrinkage-strain, hardness and optical properties as a function of reducing agent. Dent Mater. 2011; 27: 497-506.

19. Borges BC, Barreto AS, Gomes CL, Silva TR, Alves-Júnior C, Pinheiro IV, et al. Preheating of resin-based flowable materials in a microwave device: a promising approach to increasing hardness and softening resistance under cariogenic challenge. Eur J Esthet Dent. 2013; 8: 558-68.

20. Cornelio RB, Wikant A, Mjøsund H, Kopperud HM, Haasum J, Gedde UW, et al. The influence of bis-EMA vs bis-GMA on the degree of conversion and water susceptibility of experimental composite materials. Acta Odonto Scand. 2014; 72: 440-7.

21. Anagnostou M, Mountouris G, Silikas N, Kletsas D, Eliades G. Chemical, mechanical and biological properties of contemporary composite surface sealers. Dent Mater. 2015; pii: S0109-5641(15)00411-X (Epub ahead of print). 